\title{
Prolonged Cefoxitin Infusion Using Mobile Elastomeric Infusors In Outpatients With Bone And Joint Infection
}

\author{
Zoé Cavalli1,2, Agathe Becker2,3,4, Alexie Bosch2,3,4, Anne Conrad2,3,4, Claire Triffault-Filit2,3,4, Florent \\ Valour 2,3,4,5, Frederic Laurent 2,4,5,6, Sabine Cohen7, Christian Chidiac 2,3,4, Tristan Ferry 2,3,4,5凶 \\ 1. Service de Maladies Infectieuses, Hôpital de Mercy, Centre Hospitalier Régional Metz-Thionville, France \\ 2. Université Claude Bernard Lyon 1, Lyon, France \\ 3. Service de Maladies Infectieuses, Hôpital de la Croix-Rousse, Hospices Civils de Lyon, France \\ 4. Centre Interrégional de Référence des Infections Ostéo-articulaires complexes (CRIOAc Lyon), Hospices Civils de Lyon, France \\ 5. Centre International de Recherche en Infectiologie, CIRI, Inserm U1111, CNRS UMR5308, ENS de Lyon, UCBL1, Lyon, France \\ 6. Laboratoire de Bactériologie, Institut des Agents Infectieux, Hôpital de la Croix-Rousse, Hospices Civils de Lyon, France \\ 7. Laboratoire de Biochimie, Unité de pharmacologie et toxicologie, Hospices Civils de Lyon, France \\ $\square$ Corresponding author: Zoé Cavalli, Hôpital de Mercy, Centre Hospitalier Régional Metz-Thionville, 1 Allée du Château, 57245 Ars-Laquenexy, France; \\ z.cavalli@chr-metz-thionville.fr; phone +33387179256; fax +33387553588 \\ (C) Ivyspring International Publisher. This is an open access article distributed under the terms of the Creative Commons Attribution (CC BY-NC) license \\ (https://creativecommons.org/licenses/by-nc/4.0/). See http://ivyspring.com/terms for full terms and conditions.
}

Received: 2018.06.07; Accepted: 2018.07.26; Published: 2018.09.07

\begin{abstract}
We reviewed all outpatients with bone and joint infection treated with cefoxitin in continuous intravenous infusion using mobile elastomeric infusors in our regional reference center between 2014 and 2017. The stability of cefoxitin provides an interesting and well-tolerated alternative for continuous infusion in outpatients with polymicrobial bone and joint infection.
\end{abstract}

Key words: cefoxitin, infusor, bone, joint

\section{Introduction}

Cefoxitin, a second-generation cephalosporin belonging to the cephamycin group, is classically used as prophylactic antibiotherapy in surgery (1). However, its characteristics can also enable therapeutic application in bone and joint infection (BJI), and a few studies published in the 1970s showed potential efficacy in this indication (2-4). As cefoxitin is not expensive, stable for $24 \mathrm{~h}$ at $37^{\circ} \mathrm{C}$, and has an interesting spectrum targeting Staphylococcus aureus, Streptococci and Enterobacteriaceae (5), it has been increasingly prescribed using mobile elastomeric infusors in some of our outpatients with BJI. The aim of the present study was to describe this emergent practice.

\section{Methods}

A retrospective observational cohort study included all BJI outpatients treated with cefoxitin in continuous intravenous infusion using mobile elastomeric infusors (LV10 $®$ pump, Baxter (Figure 1); DOSI-FUSER ${ }^{\circledR}$, Asept Inmed; Easypump ${ }^{\circledR}$ II, Braun; or Accufuser ${ }^{\circledR}$, Vygon) in our regional reference center (Hospices Civils de Lyon, France) between 2014 and 2017. Cefoxitin was quantified in serum by liquid chromatography associated to high-resolution mass spectrometry, routinely used in the laboratory; concentrations were evaluated at steady state and were expressed as $\mathrm{mg} / \mathrm{L}$. The study was approved by the institutional review board, based on French ethical rules: informed consent waiver was granted as all data were already available. Clinical and bacteriological data were retrospectively collected from electronic medical charts used and reported as means and percentages.

\section{Results}

\section{Epidemiology}

Thirty-three patients were included (26 male: $79 \%$, with a mean age of $54.5 \pm 14.9$ years. Fifteen $(45.5 \%)$ had at least one or more underlying disease: paraplegia $(n=5)$, obesity $(n=5)$, diabetes $(n=2)$, cancer 
$(n=2)$, immunosuppressive therapy $(n=3)$ or chronic kidney disease $(n=3)$.

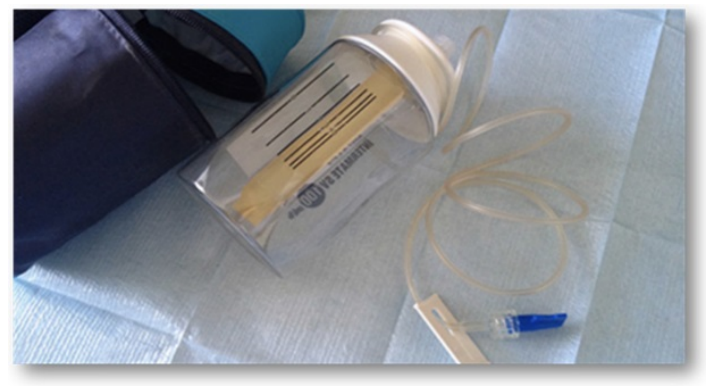

Figure 1. LV10® pump from Baxter

\section{Clinical presentation}

The BJI was localized in the lower limbs $(n=16)$, the upper limbs $(n=4)$, pelvis $(n=6)$, spine $(n=3)$, mandible $(n=3)$ or skull $(n=1)$ and consisted of medullary osteomyelitis $(n=2)$, superficial osteomyelitis $(n=14)$, localized osteomyelitis $(n=4)$, diffuse osteomyelitis $(n=7)$, prosthetic joint infection $(n=3)$ or postoperative spine infection $(n=3)$. Fifteen BJIs (45\%) were orthopedic implant-related infections. The main BJI characteristics are summarized in Table 1. Bone was exposed in 8 of the 33 patients $(24 \%)$. Infection mechanisms comprised direct inoculation (23/33: $70 \%)$, contiguity to another infection (4/33: $12 \%)$ and pressure ulcer-related osteomyelitis $(6 / 33$ : $18 \%)$. Mean BJI progression was $8.3 \pm 13.7$ years, with a median of 2 years and ranging from 1 month to 60 years. Twenty patients (64\%) had undergone surgery for the BJI before treatment with cefoxitin.

\section{Microbiology}

Bacteriological identification was available for 32 patients $(97 \%)$. BJI was polymicrobial in 23 patients $(72 \%)$, with a mean $2.8 \pm 1.9$ strains per patient and a median 3 strains: 24 (75\%) Staphylococcus spp (12 Staphylococcus aureus and 15 coagulase negative staphylococci), 8 (25\%) streptococci, 23 (72\%) anaerobic bacteria, $3(9 \%)$ enterococci and $13(41 \%)$ Enterobacteriaceae. Cefoxitin susceptibility was determined by the antimicrobial susceptibility test and interpretation following the European Committee on Antimicrobial Susceptibility Testing (EUCAST) (6). It was not possible to perform cefoxitin minimal inhibitory concentration (MIC) measurement due to the retrospective study design, as no isolates were still available.

\section{Surgery}

Thirty-two patients (97\%) underwent surgery in addition to antibiotherapy: debridement $(n=20)$, implant removal $(\mathrm{n}=10)$ including partial drop $(\mathrm{n}=1)$, negative pressure wound care $(n=5)$, debridement with implant conservation $(\mathrm{n}=6)$, internal fixation $(n=4)$, effusion drainage $(n=5)$, bone graft $(n=3)$, bone sequestrum removal $(n=4)$, cement spacer implantation $(n=2)$, one-step implant exchange $(n=1)$, or skin flap $(\mathrm{n}=1)$. The surgery was considered optimal in 24 cases (73\%). Eleven patients (33\%) required a second procedure.

\section{Antibiotherapy}

For 27 patients (82\%), other antimicrobials were already being used when cefoxitin was started. Cefoxitin was used for a mean $8.0 \pm 5.1$ weeks, within a total mean duration of antibiotherapy of 18.2 \pm 6.1 weeks. Cefoxitin was chosen by the physician because of the polymicrobial character of infection or to avoid too many oral drugs with consequent risk of non-adherence, oral intake difficulties and/or cumulative toxicity. In all patients but one, cefoxitin was used in combination: with a fluoroquinolone $(n=17)$, clindamycin $(n=10)$, rifampicin $(n=4)$, vancomycin $(n=2)$, daptomycin $(n=2)$, or metronidazole $(n=3)$. The most common dose was $6 \mathrm{~g} /$ day $(\mathrm{n}=18) ; 2$ obese patients (98 and $75 \mathrm{~kg}$ ) received $8 \mathrm{~g} /$ day, 2 obese patients (115 and $105 \mathrm{~kg}$ ) received $9 \mathrm{~g} /$ day and 1 patient with low weight (45 $\mathrm{kg}$ ) received $4 \mathrm{~g} /$ day. For all patients, cefoxitin was administered continuously using a mobile elastomeric infusor connected to a PICC-line. Cefoxitin was stopped for oral relay in 21 patients $(64 \%)$, end of antibiotherapy in $5(15 \%)$, treatment failure in $6(18 \%)$, and intolerance in $1(3 \%)$. Mean steady-state cefoxitin plasma concentration was $13.2 \pm 6.1 \mathrm{mg} / \mathrm{L}$, with a median of $11.7 \mathrm{mg} / \mathrm{L}$, ranging from 4.6 to $34 \mathrm{mg} / \mathrm{L}$, and always above the MIC of the targeted pathogen.

\section{Tolerance}

The tolerance was good for most patients $(n=30$; $91 \%)$. There were 2 minor adverse reactions; the only serious adverse event was a drug reaction with eosinophilia and systemic symptoms (DRESS), but implicating a fluoroquinolone introduced few weeks before. No patients experienced C. difficile infection.

\section{Outcome}

At the end of antibiotherapy, 23 patients (70\%) were considered cured, including 1 under suppressive therapy. Three of the 10 treatment failures related to non-optimal surgery, 5 to superinfection by cefoxitin-resistant bacteria (with bone exposure in 4 cases), 1 to non-optimal antimicrobial chemotherapy because of multiple intolerance to oral molecules; the last patient counted as failure was lost to follow-up. In cured patients, mean follow-up was $5.8 \pm 5.7$ months, with a median of 3 months. There was 1 relapse, due to a plurimicrobial osteomyelitis of the mandible 1 year after end of treatment. 
Table 1. Main characteristics of the $33 \mathrm{BJI}$

\begin{tabular}{|c|c|c|c|c|c|c|c|c|}
\hline PATIENT & $\begin{array}{l}\text { CEFOXITINE } \\
\text { DURATION } \\
\text { (WEEKS) }\end{array}$ & $\begin{array}{l}\text { CEFOXITIN } \\
\frac{\text { DAILY }}{\text { DOSE }}\end{array}$ & IMPLANT & TYPES OF BJI & $\underline{\text { SURGERY }}$ & MICROBIOLOGY & $\begin{array}{l}\text { ADDITIONAL MOLECULE TO } \\
\text { IV CEFOXITIN }\end{array}$ & OUTCOME \\
\hline 1 & 10 & $9 g$ & yes & $\begin{array}{l}\text { post-operative } \\
\text { spine infection }\end{array}$ & $\begin{array}{l}\text { collection drainage, } \\
\text { partial implant removal }\end{array}$ & Finegoldia, E. coli & Ciprofloxacin Daptomycin & cure \\
\hline 2 & 4 & $6 \mathrm{~g}$ & no & $\begin{array}{l}\text { superficial } \\
\text { osteomyelitis }\end{array}$ & none & $\begin{array}{l}\text { S. epidermidis, } \\
\text { Peptostreptococcus }\end{array}$ & Ciprofloxacin & failure \\
\hline 3 & 4 & $6 g$ & yes & $\begin{array}{l}\text { diffuse } \\
\text { osteomyelitis }\end{array}$ & $\begin{array}{l}\text { implant removal, } \\
\text { debridement }\end{array}$ & $\begin{array}{l}\text { S. aureus, } S . \\
\text { lugdunensis, } \\
\text { Peptostreptococcus }\end{array}$ & Clindamycin & cure \\
\hline 4 & 5 & $6 \mathrm{~g}$ & yes & $\begin{array}{l}\text { diffuse } \\
\text { osteomyelitis }\end{array}$ & $\begin{array}{l}\text { debridement, implant } \\
\text { removal, osteosynthesis, } \\
\text { bone graft }\end{array}$ & S. capitis & Ofloxacin & failure \\
\hline 5 & 12 & $8 g$ & yes & $\begin{array}{l}\text { prosthetic joint } \\
\text { infection }\end{array}$ & $\begin{array}{l}\text { collection drainage, } \\
\text { debridement and implant } \\
\text { retention }\end{array}$ & $\begin{array}{l}\text { Streptococcus } \\
\text { mitis/oralis }\end{array}$ & Levofloxacin & failure \\
\hline 6 & 2 & $6 \mathrm{~g}$ & no & $\begin{array}{l}\text { superficial } \\
\text { osteomyelitis }\end{array}$ & $\begin{array}{l}\text { debridement, negative } \\
\text { pressure wound care } \\
\text { establishment }\end{array}$ & S. aureus & Ofloxacin & failure \\
\hline 7 & 6 & $6 \mathrm{~g}$ & no & $\begin{array}{l}\text { localized } \\
\text { osteomyelitis }\end{array}$ & $\begin{array}{l}\text { debridement, bone } \\
\text { sequestrum removal }\end{array}$ & $\begin{array}{l}\text { S. aureus, } \\
\text { Streptococcus } \\
\text { constellatus, } \\
\text { Bacteroides, } \\
\text { Citrobacter }\end{array}$ & Ofloxacin Metronidazole & cure \\
\hline 8 & 4 & $6 \mathrm{~g}$ & yes & $\begin{array}{l}\text { superficial } \\
\text { osteomyelitis }\end{array}$ & $\begin{array}{l}\text { debridement, implant } \\
\text { removal, negative } \\
\text { pressure wound care } \\
\text { establishment }\end{array}$ & $\begin{array}{l}\text { S. aureus, } S \text {. } \\
\text { lugdunensis, } \\
\text { Corynebacterium, E. } \\
\text { coli }\end{array}$ & Ofloxacin & failure \\
\hline 9 & 2 & $6 \mathrm{~g}$ & yes & $\begin{array}{l}\text { post-operative } \\
\text { spine infection }\end{array}$ & $\begin{array}{l}\text { debridement and implant } \\
\text { retention }\end{array}$ & $\begin{array}{l}\text { S. capitis, } \\
\text { Propionibacterium, E. } \\
\text { coli, Proteus }\end{array}$ & Ofloxacin & cure \\
\hline 10 & 8 & $6 \mathrm{~g}$ & yes & $\begin{array}{l}\text { superficial } \\
\text { osteomyelitis }\end{array}$ & $\begin{array}{l}\text { implant removal, } \\
\text { negative pressure wound } \\
\text { care establishment }\end{array}$ & $\begin{array}{l}\text { S. aureus, } \\
\text { Enterococcus avium, } \\
\text { Helcococcus, Proteus, } \\
\text { E. coli }\end{array}$ & Teicoplanin & cure \\
\hline 11 & 8 & $6 \mathrm{~g}$ & yes & $\begin{array}{l}\text { diffuse } \\
\text { osteomyelitis }\end{array}$ & $\begin{array}{l}\text { implant removal, } \\
\text { osteosynthesis, bone graft }\end{array}$ & $\begin{array}{l}\text { S. capitis, } \\
\text { Streptococcus } \\
\text { mitis/parasanguinis }\end{array}$ & Ofloxacin & cure \\
\hline 12 & 6 & $6 \mathrm{~g}$ & no & $\begin{array}{l}\text { superficial } \\
\text { osteomyelitis }\end{array}$ & $\begin{array}{l}\text { debridement, bone } \\
\text { sequestrum removal }\end{array}$ & $\begin{array}{l}\text { S. aureus, Prevotella, } \\
\text { Haemophilus }\end{array}$ & Clindamycin & cure \\
\hline 13 & 6 & $6 g$ & no & $\begin{array}{l}\text { localized } \\
\text { osteomyelitis }\end{array}$ & debridement & $\begin{array}{l}\text { Streptococcus } \\
\text { parasanguinis/mitis/sa } \\
\text { livarius, Veilonella, } \\
\text { Citrobacter }\end{array}$ & Levofloxacin Metronidazole & cure \\
\hline 14 & 6 & $9 g$ & no & $\begin{array}{l}\text { superficial } \\
\text { osteomyelitis }\end{array}$ & debridement & $\begin{array}{l}\text { Propionibacterium, } \\
\text { Gemella, } \\
\text { Fusobacterium, } \\
\text { Finegoldia }\end{array}$ & Metronidazole & cure \\
\hline 15 & 23 & $6 g$ & yes & $\begin{array}{l}\text { superficial } \\
\text { osteomyelitis }\end{array}$ & $\begin{array}{l}\text { debridement and implant } \\
\text { retention, skin flap }\end{array}$ & $\begin{array}{l}\text { S. aureus, S. } \\
\text { lugdunensis, } \\
\text { Streptococcus mitis, } \\
\text { Peptinophilus, } \\
\text { Finegoldia }\end{array}$ & Clindamycin & cure \\
\hline 16 & 3 & $6 g$ & no & $\begin{array}{l}\text { superficial } \\
\text { osteomyelitis }\end{array}$ & debridement & S. aureus & Clindamycin & cure \\
\hline 17 & 12 & $6 \mathrm{~g}$ & yes & $\begin{array}{l}\text { superficial } \\
\text { osteomyelitis }\end{array}$ & $\begin{array}{l}\text { debridement and implant } \\
\text { retention }\end{array}$ & $\begin{array}{l}\text { S. aureus, } \\
\text { Streptococcus } \\
\text { agalactiae }\end{array}$ & Ofloxacin Rifampicin & cure \\
\hline 18 & 12 & $4 \mathrm{~g}$ & no & $\begin{array}{l}\text { diffuse } \\
\text { osteomyelitis }\end{array}$ & $\begin{array}{l}\text { debridement, bone graft, } \\
\text { osteosynthesis }\end{array}$ & sterile & Ciprofloxacin & cure \\
\hline 19 & 24 & $6 \mathrm{~g}$ & yes & $\begin{array}{l}\text { superficial } \\
\text { osteomyelitis }\end{array}$ & $\begin{array}{l}\text { debridement, } \\
\text { debridement and implant } \\
\text { retention }\end{array}$ & $\begin{array}{l}\text { Enterococcus faecalis, } \\
\text { Prevotella, } \\
\text { Peptinophilus, } \\
\text { Porphyromonas, E. } \\
\text { coli }\end{array}$ & Daptomycin Ciprofloxacin & cure \\
\hline 20 & 12 & $6 \mathrm{~g}$ & no & $\begin{array}{l}\text { superficial } \\
\text { osteomyelitis }\end{array}$ & $\begin{array}{l}\text { collection drainage, } \\
\text { debridement }\end{array}$ & $\begin{array}{l}\text { S. aureus, } \\
\text { Streptococcus } \\
\text { anginosus/constellatus } \\
\text { /interm, Bacteroides, } \\
\text { Actinomyces, } \\
\text { Corynebacterium, } \\
\text { Finegoldia, E. coli }\end{array}$ & Clindamycin & failure \\
\hline 21 & 6 & $6 \mathrm{~g}$ & no & $\begin{array}{l}\text { medullary } \\
\text { osteomyelitis }\end{array}$ & $\begin{array}{l}\text { collection drainage, } \\
\text { debridement }\end{array}$ & S. aureus & Rifampicin & cure \\
\hline 22 & 12 & $6 g$ & yes & $\begin{array}{l}\text { diffuse } \\
\text { osteomyelitis }\end{array}$ & $\begin{array}{l}\text { implant removal, } \\
\text { debridement }\end{array}$ & S. aureus & Clindamycin & failure \\
\hline 23 & 8 & $6 g$ & no & $\begin{array}{l}\text { superficial } \\
\text { osteomyelitis }\end{array}$ & $\begin{array}{l}\text { debridment, negative } \\
\text { pressure wound care } \\
\text { establishment }\end{array}$ & $\begin{array}{l}\text { S. capitis, } \\
\text { Propionibacterium, } \\
\text { Proteus }\end{array}$ & Moxifloxacin & failure \\
\hline 24 & 8 & $6 \mathrm{~g}$ & yes & $\begin{array}{l}\text { prosthetic joint } \\
\text { infection }\end{array}$ & $\begin{array}{l}\text { debridement and partial } \\
\text { implant retention, partial }\end{array}$ & $\begin{array}{l}\text { S. lugdunensis, } S \text {. } \\
\text { caprae, S. capitis }\end{array}$ & Ofloxacin & cure \\
\hline
\end{tabular}




\begin{tabular}{|c|c|c|c|c|c|c|c|c|}
\hline PATIENT & 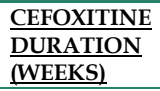 & $\frac{\text { CEFOXITIN }}{\underline{\text { DAILY }}}$ & IMPLANT & TYPES OF BJI & $\underline{\text { SURGERY }}$ & MICROBIOLOGY & $\begin{array}{l}\text { ADDITIONAL MOLECULE TO } \\
\text { IV CEFOXITIN }\end{array}$ & OUTCOME \\
\hline & & & & & $\begin{array}{l}\text { implant one-stage } \\
\text { exchange }\end{array}$ & & & \\
\hline 25 & 6 & $6 \mathrm{~g}$ & no & $\begin{array}{l}\text { superficial } \\
\text { osteomyelitis }\end{array}$ & debridement & $\begin{array}{l}\text { S. aureus, } \\
\text { Actinomyces }\end{array}$ & Clindamycin & cure \\
\hline 26 & 3 & $6 \mathrm{~g}$ & no & $\begin{array}{l}\text { localized } \\
\text { osteomyelitis }\end{array}$ & $\begin{array}{l}\text { debridement, bone } \\
\text { sequestrum removal }\end{array}$ & $\begin{array}{l}\text { Streptococcus } \\
\text { gordoni/mitis/constella } \\
\text { tus/intermedius, } \\
\text { Actinomyces, } \\
\text { Haemophilus }\end{array}$ & no & cure \\
\hline 27 & 6 & $6 \mathrm{~g}$ & no & $\begin{array}{l}\text { localized } \\
\text { osteomyelitis }\end{array}$ & debridement & Veilonella & Clindamycin & cure \\
\hline 28 & 6 & $6 g$ & yes & $\begin{array}{l}\text { diffuse } \\
\text { osteomyelitis }\end{array}$ & $\begin{array}{l}\text { implant removal, } \\
\text { debridement }\end{array}$ & $\begin{array}{l}\text { S. aureus, } \\
\text { Propionibacterium }\end{array}$ & Clindamycin & cure \\
\hline 29 & 8 & $6 \mathrm{~g}$ & yes & $\begin{array}{l}\text { medullary } \\
\text { osteomyelitis }\end{array}$ & $\begin{array}{l}\text { collection drainage, } \\
\text { implant removal, } \\
\text { negative pressure wound } \\
\text { care establishment }\end{array}$ & $\begin{array}{l}\text { S. epidermidis, } \\
\text { Corynebacterium }\end{array}$ & Rifampicin & failure \\
\hline 30 & 12 & $6 \mathrm{~g}$ & no & $\begin{array}{l}\text { superficial } \\
\text { osteomyelitis }\end{array}$ & debridement & $\begin{array}{l}\text { S. aureus, } \\
\text { Streptococcus } \\
\text { equismilis, } \\
\text { Corynebacterium, } \\
\text { Proteus }\end{array}$ & Vancomycin Ofloxacine & cure \\
\hline 31 & 3 & $6 g$ & no & $\begin{array}{l}\text { post-operative } \\
\text { spine infection }\end{array}$ & debridement & S. epidermidis & Clindamycin & cure \\
\hline 32 & 8 & $8 g$ & yes & $\begin{array}{l}\text { prosthetic joint } \\
\text { infection }\end{array}$ & $\begin{array}{l}\text { implant removal, cement } \\
\text { spacer establishment }\end{array}$ & Proteus & Ofloxacin & cure \\
\hline 33 & 8 & $6 \mathrm{~g}$ & no & $\begin{array}{l}\text { diffuse } \\
\text { osteomyelitis }\end{array}$ & $\begin{array}{l}\text { debridement, cement } \\
\text { spacer establishment, } \\
\text { osteosynthesis }\end{array}$ & $\begin{array}{l}\text { S. capitis, } S \text {. } \\
\text { epidermidis, } \\
\text { Propionibacterium }\end{array}$ & Rifampicin & failure \\
\hline
\end{tabular}

\section{Discussion}

$\mathrm{BJI}$ is difficult to manage, often requiring surgery and prolonged antibiotherapy, sometimes with several drugs that may be poorly tolerated. Cefoxitin is an old antibiotic developed in the early 1970s, but not available in all countries; it is mostly used in prophylactic treatment, but has specific features that allow increasing use in curative treatment of BJI (7).

Few studies of cefoxitin in BJI have been published. Bone diffusion is similar to that of other cephalosporins, reaching $20 \%$ of serum level in bone and synovial fluid 1 hour after administration $(8,9)$. Perkins et al. reported 27 skin and soft tissue infections treated by cefoxitin, with $93 \%$ success, including 3 with contiguous osteomyelitis (4). More interestingly, Schurman et al. reported 31 patients with acute or chronic infections of bone, joint or muscle and tendon, with an $84 \%$ cure rate (2).

Cefoxitin is a broad-spectrum molecule, including gram-positive cocci (methicillin-susceptible staphylococci, streptococci), Gram-negative bacilli (including extended-spectrum beta-lactamases [ESBL] producing Enterobacteriaceae) and anaerobic bacteria (5). In comparison, ceftriaxone exhibits sub-optimal in-vitro activity against MSSA isolates and is not active on anaerobes, unlike cefoxitin, which is usually active on Bacteroides fragilis (10). In the present study, cefoxitin was chosen mainly because of the nature of the BJI, so as to avoid using 3 or 4 oral antibiotics, with potentially higher risk of cumulative toxicity.

A maximum time above 2-3 target bacterium MIC is generally considered a suitable pharmacologic goal. Considering that, for susceptible isolates, the maximum MIC of cefoxitin is $8 \mathrm{mg} / \mathrm{L}$ for Gram-negative bacilli, $4 \mathrm{mg} / \mathrm{L}$ for $S$. aureus and $S$. lugdunensis and $8 \mathrm{mg} / \mathrm{L}$ for $S$. saprophyticus (6), the present mean steady-state level of $13.2 \mathrm{mg} / \mathrm{L}$ reached this therapeutic target. These concentrations were obtained with a mean dose of $6 \mathrm{~g} /$ day, adjusted for patients with extreme weights.

Cefoxitin is also a time-dependent antibiotic, stable at room temperature $(11,12)$ and at $37^{\circ} \mathrm{C}(13)$. Consequently, continuous infusion administered at home with elastometric diffusors is a very interesting means of reducing hospital stay. Elastomeric infusors in polyisoprene are preferable than silicone for constant stable infusion (14). Cefoxitin stability in elastometric diffusors was evaluated by Baxter, but only up to $8^{\circ} \mathrm{C}$ (manufacturer's data). However, the mean pharmacological dose at equilibrium was adequate in the present patients.

Finally, adverse effects of cefoxitin are rare. Cross-reactivity between cephamycins and other beta-lactams was reported, but incidence of allergic reaction was low and any reactions were mild (15). Other possible adverse events comprise: local reactions, and gastro-intestinal, hematologic, hepatic or renal disorders (5). The seminal study published in 1977, with 143 patients, found a 1.4\% rate of eruption, $2 \%$ cytolysis, $2 \%$ leucopenia, $2.5 \%$ eosinophilia and $5 \%$ thrombophlebitis (16). In the present study, global tolerance was good, with only 1 severe adverse event, which was likely not related to cefoxitin but to another antibiotic used concomitantly. 


\section{Conclusion}

Cefoxitin can be a useful outpatient parenteral alternative in the treatment of BJI. Its spectrum is interesting in case of polymicrobial infection, its potential stability enables continuous infusion with elastomeric infusors, and tolerance is generally good. A prospective study with homogeneous infusor management (type of diffusor, dilution, stability analysis) in patients with BJI and with blood pharmacokinetic/ pharmacodynamic analysis is needed to confirm these results.

\section{Competing Interests}

The authors have declared that no competing interest exists.

\section{References}

1. [Internet] American Society of Health-System Pharmacists. Clinical Practice Guidelines for Antimicrobial Prophylaxis in Surgery. 2013. Available from: https://www.ashp.org/-/media/assets/policy-guidelines/docs/therapeutic -guidelines/therapeutic-guidelines-antimicrobial-prophylaxis-surgery.ashx?1 $\mathrm{a}=$ en\&hash=A15B4714417A51A03E5BDCAC150B94EAF899D49B

2. Schurman DJ, Dillingham M. Clinical evaluation of cefoxitin in treatment of infections in 47 orthopedic patients. Rev Infect Dis. 1979 Feb;1(1):206-9.

3. Webb D, Thadepalli H, Bach V, Roy I. Clinical and experimental evaluation of cefoxitin therapy. Chemotherapy. 1979;25(4):233-42.

4. Perkins RL, Slama TG, Fass RJ, Prior RB, Plouffe JF, Warner JF, et al. Therapy of skin, soft tissue, and bone infections with cefoxitin sodium. Rev Infect Dis. 1979 Feb;1(1):165-9.

5. [Internet] Cefoxitin - FDA prescribing information, side effects and uses. Drugs.com. Available from: https://www.drugs.com/pro/cefoxitin.html

6. [Internet] European Committee on Antimicrobial Susceptibility Testing. The European Committee on Antimicrobial Susceptibility Testing. Breakpoint tables for interpretation of MICs and zone diameters. Version 8.0. 2018. Available from: http://www.eucast.org.

7. Miller AK, Celozzi E, Kong Y, Pelak BA, Hendlin D, Stapley EO. Cefoxitin, a semisynthetic cephamycin antibiotic: in vivo evaluation. Antimicrob Agents Chemother. 1974 Jan;5(1):33-7.

8. Schurman DJ, Burton DS, Kajiyama G. Cefoxitin antibiotic concentration in bone and synovial fluid. Clin Orthop. 1982 Aug;(168):64-8.

9. Summersgill JT, Schupp LG, Raff MJ. Comparative penetration of metronidazole, clindamycin, chloramphenicol, cefoxitin, ticarcillin, and moxalactam into bone. Antimicrob Agents Chemother. 1982 Apr;21(4):601-3.

10. Bremmer DN, Balada-Llasat J-M, Goff DA, Bauer KA. Ceftriaxone Etest non-susceptible methicillin susceptible Staphylococcus aureus time-kill responses. Diagn Microbiol Infect Dis. 2017 Jun;88(2):192-4.

11. Bosso JA, Townsend RJ. Stability of clindamycin phosphate and ceftizoxime sodium, cefoxitin sodium, cefamandole nafate, or cefazolin sodium in two intravenous solutions. Am J Hosp Pharm. 1985 Oct;42(10):2211-4.

12. Das Gupta V, Stewart KR. Stability of cefamandole nafate and cefoxitin sodium solutions. Am J Hosp Pharm. 1981 Jun;38(6):875-9.

13. Stiles ML, Tu YH, Allen LV. Stability of cefazolin sodium, cefoxitin sodium, ceftazidime, and penicillin $\mathrm{G}$ sodium in portable pump reservoirs. Am J Hosp Pharm. 1989 Jul;46(7):1408-12.

14. Guiffant G, Durussel J-J, Flaud P, Vigier J-P, Dupont C, Bourget P, et al. Mechanical performances of elastomers used in diffusers. Med Devices Auckl NZ. 2011;4:71-6.

15. Crotty DJ, Chen XJC, Scipione MR, Dubrovskaya Y, Louie E, Ladapo JA, et al. Allergic Reactions in Hospitalized Patients With a Self-Reported Penicillin Allergy Who Receive a Cephalosporin or Meropenem. J Pharm Pract. 2017 Feb;30(1):42-8.

16. McCloskey RV. Results of a clinical trial of cefoxitin, a new cephamycin antibiotic. Antimicrob Agents Chemother. 1977 Nov;12(5):636-41. 